Ciencia de los alimentos/Food Science

Cienc Tecn UTEQ (2017) 10(1) p 41-46 ISSN 1390-4051; e-ISSN 1390-4043 이요 (1)

\title{
Potencial antifúngico de Citrus sinensis y Citrus nobilis sobre el crecimiento de Rhizopus stolonifer y Colletotrichum gloeosporioides en papaya
}

\section{Antifungal potential of Citrus sinensis and Citrus nobilis on the Rhizopus stolonifer and Colletotrichum gloeosporioides growth in papaya}

Flor Jajaira Narváez Baque ${ }^{1},{ }^{\bullet}$ Sonnia Esther Barzola Miranda ${ }^{1}$, Flor Marina Fon-Fay Vásquez ${ }^{1}$, Malena Jacqueline Martínez Chávez ${ }^{1}$ Juan Alejandro Neira Mosquera ${ }^{1,2}$, Sungey Naynee Sánchez Llaguno ${ }^{2}$

${ }^{1}$ Universidad Técnica Estatal de Quevedo. Facultad de Ciencias de la Ingeniería, Carrera de Ingeniería Agroindustrial. Campus Manuel Haz Álvarez. Av. Quito Km 1.5 vía Santo Domingo de los Tsáchilas.EC.120301.Quevedo,Ecuador. florjbaq.narvaez@uteq.edu.ec; `sbarzola@uteq.edu.ec; ffonfay@uteq.edu.ec; mmartinez@uteq.edu.ec; neiramosquera@uteq.edu.ec

${ }^{2}$ Universidad de las Fuerzas Armadas-ESPE, Departamento de Ciencias de la Vida. Av. General Rumiñahui $s / n$ Sangolqui, Ecuador, P.O.BOX: 171-5-231B. snsanchez@espe.edu.ec

Rec.: 22.06.2016. Acept.: 27.12.2016.

Publicado el 1 de junio de 2017

\section{Resumen}

$\mathrm{E}$ n este estudio se evalúo el efecto antifúngico de los aceites esenciales de hojas y pericarpio de Citrus sinensis (Naranja) y Citrus nobilis (Mandarina) sobre el crecimiento de los hongos Rhizopus stolonifer y Colletotrichum gloeosporioides aislados de papaya Maradol. Se aplicó un análisis estadístico con un arreglo factorial de bloques $\mathrm{A}(2)$ $\mathrm{xB}(2) \mathrm{xC}(3)$ con 2 repeticiones considerando como Factor $\mathrm{A}$ : Aceites esenciales (Citrus sinensis y Citrus nobilis), Factor B: Origen de los aceites esenciales (hojas y pericarpio), Factor $\mathrm{C}$ : Concentraciones $(1 \%, 2 \%$ y $4 \%)$; se evaluó durante siete días el crecimiento radial de los hongos y posteriormente se realizó el conteo de esporas en la cámara de Neubauer. Para el análisis de datos se empleó el paquete estadístico StatGraphics; para la separación de medias de los niveles de los tratamientos se realizó la prueba de significación Tukey $(p<0.05)$. Los mejores resultados, tanto para la inhibición de crecimiento radial, como de esporas fueron el aceite esencial de Citrus sinensis (a0), obtenido de las hojas (b0) y con 4\% de concentración de aceite en la solución empleada (c2) en los dos microorganismos aplicados: Rhizopus stolonifer y Colletorichum gloeosporioides.

Palabras clave: aceites esenciales, hongos, papaya, inhibición radial, esporas.

\begin{abstract}
$\mathrm{T}$ his study evaluated the antifungal effect of essential oils obtained from Citrus sinensis (orange) and Citrus nobilis (mandarine) leaves and pericarp on the growth of fungi (Rhizopus stolonifer and Colletotrichum gloeosporioides) isolated from papaya Maradol. A statistical analysis with a factorial block arrangement $\mathrm{A}(2) \times \mathrm{B}(2) \times \mathrm{C}(3)$ with two repetitions was made, considering: Factor A: essential oils (Citrus sinensis and Citrus nobilis), Factor B: essential oil source (leaves and pericarp), Factor C: Concentrations $(1 \%, 2 \%$ y $4 \%)$. During seven days fungi radial growth was evaluated, and after this spore counting was carried out in the Neubauer chamber (haemocytometer). For data analysis StatGraphics was used and for determining the treatments' level means, significance Tukey test $(\mathrm{p}<0.05)$ was made. The best results inhibiting radial growth, and spores were: essential oil from Citrus sinensis (a0), obtained from the leaves (b0) with $4 \%$ oil concentration in the solution used (c2) in the microorganisms Rhizopus stolonifer and Colletorichum gloeosporioides.
\end{abstract}

Key words: essential oils, fungi, papaya, radial inhibition, spores. 


\section{Introducción}

L as frutas tropicales como la papaya tiene gran importancia económica por su alto valor nutritivo, propiedades sensoriales y medicinales, características que han contribuido a incrementar su cultivo, siendo una fruta susceptible al manejo postcosecha, requiere cuidados para evitar pérdidas y mermas de un alto costo económico (Suárez et al., 2013). Su valor nutritivo comprende un adecuado balance de compuestos orgánicos e inorgánicos tales como carbohidratos, vitaminas A, B, C, K, ácido fólico y minerales como el calcio, fosforo, magnesio, hierro, sodio, potasio, entre otros (Rodríguez y Veneros, 2011).

Después de cosechados, los frutos pasan por una serie de transformaciones endógenas resultantes del metabolismo celular, el aumento de los azúcares solubles, de agua libre y de las pectinas es acompañado por la reducción de algunos componentes fenólicos y protopectínicos, que tornan los frutos más sensibles al daño mecánico y al ataque de diversos microorganismos (Mendoza et al., 2013). La maduración de la papaya ocurre inmediatamente después de la cosecha por lo tanto se recomienda conservarse a temperaturas entre 8 a 10 ${ }^{\circ} \mathrm{C}$ por un periodo máximo de 4 semanas; y de 5 a 7 días si la temperatura es de $22{ }^{\circ} \mathrm{C}$; sin embargo, durante este tiempo es susceptible al ataque de microorganismos patógenos, siendo los hongos Colletotrichum sp., Rhizopus stolonifer, Botrytis cinerea, Penicillium italicum y Penicillium digitatum, los causantes de las enfermedades de postcosecha más comunes (Guédez et al., 2014).

Una de las alternativas que se han estudiado con resultados prometedores, se basa en el hecho que, las plantas elaboran metabolitos secundarios con la finalidad de disminuir el ataque de parásitos y depredadores naturales, muchos de estos compuestos se caracterizan por ser inocuos para el ser humano, y se consideran como "fungicidas naturales" (Landero et al., 2013). Por tanto, se incrementó el interés de su aplicación para el manejo de enfermedades en campo y poscosecha, debido al efecto que exhiben sobre los fitopatógenos (Duarte et al., 2013).

Este estudio evaluó el potencial antifúngico de los aceites esenciales de Citrus sinensis (naranja) y Citrus nobilis (mandarina) aplicado en cepas de Rhizopus stolonifer y Colletotrichum gloeosporioides, aisladas del mesocarpio de la papaya de variedad "Maradol" colectada en el cantón Valencia, sector agrícola de la provincia de Los Ríos, Ecuador.

\section{Materiales y métodos}

\section{Obtención del material vegetal}

En el Cantón Valencia, Provincia de Los Ríos, Ecuador. se obtuvieron las papayas de la variedad "Maradol".

\section{Aislamiento de Rhizopus stolonifer y Colletotrichum gloeosporioides}

El material vegetal (papaya) fue previamente lavado con solución de hipoclorito de sodio al 3\%. Posteriormente, con agua destilada estéril se enjuagó tres veces consecutivas. Seguidamente, se seleccionaron 5 muestras del mesocarpio de $0.50 \mathrm{~cm}^{2}$ entre las partes intermedias del tejido sano y parte del tejido en descomposición; fueron transferidas al medio de cultivo agar papa dextrosa (APD) (Neogen corporation) y se aplicó Sulfato de estreptomicina ${ }^{\circledR}$ al $1 \%$. Para el aislamiento de los microorganismos se incubaron las placas a una temperatura de $24{ }^{\circ} \mathrm{C}$ por 7 días. Todo el procedimiento se desarrolló en cámara de flujo laminar cumpliendo las condiciones de asepsia y esterilidad requeridas (Castellanos et al., 2011).

\section{Identificación de colonias}

Cada colonia desarrollada se separó y purificó por el sistema de repiques, usando medio de cultivo APD y la observación se efectuó por montaje microscópico. La identificación a nivel de género se realizó siguiendo las claves de Barnett y Hunter (1987), esto permitió obtener colonias aisladas de Rhizopus stolonifer y Colletotrichum gloeosporioides. La identificación se hizo tomando en cuenta las características del micelio, el color de la colonia, la forma de conidióforos; forma, tamaño y color de los conidios (Von, 1981; Barnett y Hunter, 1987).

\section{Obtención de aceites esenciales}

Los aceites esenciales de pericarpio y hojas de Citrus sinensis y Citrus nobilis fueron extraidos y obtenidos mediante hidrodestilación. Los aceites de los pericarpios fueron preparados en el Laboratorio IsabrukBotanik S.A. ubicado en la ciudad de Ambato, provincia de Tungurahua, Ecuador. Mientras, los aceites de las hojas se obtuvieron en el laboratorio de Química Básica, UTEQ-Quevedo, provincia de Los Ríos, Ecuador.

\section{Determinación de la capacidad fungicida}

El efecto inhibitorio de los aceites esenciales sobre los hongos Rhizopus stolonifer y Colletotrichum gloeosporioides se determinó mediante la observación del crecimiento radial del micelio, medido en mm y conteo del número de esporas por $\mathrm{mL}$ de solución (Castellanos et al., 2011).

\section{Determinación del crecimiento radial}

La siembra se realizó en cajas de petri previamente esterilizadas con medio APD, a estas se agregó concentraciones de aceites de $1 \%, 2 \%$ y $4 \%$ equivalentes a $100 \mu \mathrm{L}, 200 \mu \mathrm{L}$ y $400 \mu \mathrm{L}$. Previamente, los aceites se mezclaron con tween 20 a una concentración de $1 \%$ para mejorar su solubilidad y estabilidad en el medio. Cada concentración y tipo de aceite se colocó en placas por separado. Una vez homogenizado y solidificado el medio, una muestra de aproximadamente $6 \mathrm{~mm}$ 
de diámetro de cada hongo, se colocó sobre el agar. El ensayo se realizó por duplicado y se incluyó una muestra (testigo) únicamente con el medio APD y tween 20 a concentración de $1 \%$. Las placas se incubaron a $24{ }^{\circ} \mathrm{C}$ y se registro los datos cada 24 horas y durante 7 días, con mediciones del crecimiento radial en mm usando un calibrador vernier. Para determinar el porcentaje de inhibición se usó la formula ( $\mathrm{T}$ $\operatorname{Tr} / \mathrm{T}$ )* 100, donde: T: testigo y Tr: tratamiento.

\section{Conteo de esporas}

Una vez observado el crecimiento radial se contó el número de esporas por microorganismo ensayado. A cada una de las placas se adicionó $10 \mathrm{~mL}$ de agua destilada estéril, cada placa se agitó con una varilla de vidrio y se tomó una alícuota de $1 \mathrm{~mL}$ de agua; se agregó $1 \mu \mathrm{L}$ de tween 20 al 1\%, las muestras se colocaron en viales Eppendorf y se agitaron en un Vortex mixer (Labnet SO200) para obtener una solución homogénea. Se contó el número de esporas, y se usó una cámara de Neubauer (Marienfeld $0.0025 \mathrm{~mm}$ x $0.10 \mathrm{~mm}$ de profundidad) y un microscopio (Olympus CX1, lente número 10). El porcentaje de inhibición se determinó mediante la fórmula $(\mathrm{T}-\mathrm{Tr} / \mathrm{T}) * 100$, donde: $\mathrm{T}$ : testigo y $\mathrm{Tr}$ : tratamiento.

Cuadro 1. Tratamientos estudiados en la valoración de los aceites esenciales de Citrus sinensis (naranja) y Citrus nobilis (mandarina) en la inhibición de hongos de papaya

\begin{tabular}{lll}
\hline Trat. & Simb. & Descripción \\
\hline T1 & $a_{0} b_{0} c_{0}$ & Naranja, Hojas, 1\% \\
T2 & $a_{0} b_{0} c_{1}$ & Naranja, Hojas, 2\% \\
T3 & $a_{0} b_{0} c_{2}$ & Naranja, Hojas, 4\% \\
T4 & $a_{0} b_{1} c_{0}$ & Naranja, Corteza del fruto, 1\% \\
T5 & $a_{0} b_{1} c_{1}$ & Naranja, Corteza del fruto, 2\% \\
T6 & $a_{0} b_{1} c_{2}$ & Naranja, Corteza del fruto, 4\% \\
T7 & $a_{1} b_{0} c_{0}$ & Mandarina, Hojas, 1\% \\
T8 & $a_{1} b_{0} c_{1}$ & Mandarina, Hojas, 2\% \\
T9 & $a_{1} b_{0} c_{2}$ & Mandarina, Hojas, 4\% \\
T10 & $a_{1} b_{1} c_{0}$ & Mandarina, Corteza del fruto, 1\% \\
T11 & $a_{1} b_{1} c_{1}$ & Mandarina, Corteza del fruto, 2\% \\
T12 & $a_{1} b_{1} c_{2}$ & Mandarina, Corteza del fruto, 4\% \\
\hline
\end{tabular}

\section{Análisis estadísticos}

Se aplicó un ANOVA, con diseño de bloques al azar mediante arreglo factorial $\mathrm{A}^{*} \mathrm{~B} * \mathrm{C}$ con tres replicas (Cuadro 1); Los niveles estudiados por tratamiento fueron: Factor A: Aceites esenciales (naranja y mandarina), Factor B: Origen de los aceites esenciales (hojas y pericarpio) y el Factor C: concentraciones $(1 \%, 2 \%$ y $4 \%)$, la tabulación de resultados se realizó mediante el software Stats Graphics Centurión de la Universidad de Massachusetts. Para la separación de medias de los tratamientos se realizó mediante Tukey $(\mathrm{p}<0.05)$.

\section{Resultados y discusión}

$\mathrm{E}$ la capacidad fungica, las interacciones $\left(\mathrm{A}^{*} \mathrm{~B} * \mathrm{C}\right)$ para la inhibición del crecimiento radial del hongo $R$. stolonifer, los tratamientos evitaron $100 \%$ de proliferación, a diferencia del alblc0 (Citrus nobilis, corteza del fruto, 1\%) y alblc1 (Citrus nobilis, corteza del fruto, 2\%) (Cuadro 2), resultados que coinciden con lo reportado por Ronquillo (2007) en su estudio de evaluación del potencial antimicrobiano de películas comestibles con aceites esenciales in vitro e in situ. Mientras, en la inhibición de esporas, se observó 100\% en los tratamientos a0b0c1 (Citrus sinensis, Hojas, 2\%), a0b0c2 (Citrus sinensis, Hojas, 4\%), alb0c2 (Citrus nobilis, Hojas, 4\%), a0b0c0 (Citrus sinensis, Hojas, 1\%), alb0c0 (Citrus nobilis, Hojas, 1\%) superiores a los reportados por Tzortzakis y Economakis (2007) en su investigación sobre actividad anti fúngica del aceite esencial de Lemongrass (Cymbopogon citratus L.) contra hongos patógenos de poscosecha (70\%). Por tanto, se puede expresar que los niveles de inhibición son buenos, y la variación podría estar sujeta a factores intrínsecos de la fruta utilizada o a su vez a factores ambientales.

Asimismo, C. gloeosporioides, tuvo la misma tendencia que $R$. stolonifer, efectuando una inhibición del 100\% de crecimiento radial en los mismos tratamientos (Cuadro 2), superiores a los reportados por Pineda et al. (2010) en su estudio de propiedad fungistática in vitro de propóleos sobre tres aislamientos de Colletotrichum gloeosporioides (30\%); similares resultados se obtuvo en inhibición de esporas (100\% de inhibición), valores superiores a los reportados por Silva et al. (2011) en su investigación titulada: Inhibición del crecimiento micelial y la germinación Colletotrichum gloeosporioides aplicando aceite de neem (Azadirachta indica) que fue solo del $61 \%$.

El aceite esencial de Citrus sinensis reveló una fuerte actividad inhibitoria tanto en crecimiento radial como en número de esporas de hongos para $R$. stolonifer (Cuadro 3). Estos resultados son similares a los obtenidos por Guédez et al. (2014) quienes evaluaron el aceite Citrus sinensis en frutos de lechosa (Carica papaya L.) en la postcosecha, consiguiendo $100 \%$ de inhibición.

En la inhibición de esporas se evidenció $97.06 \%$ con el aceite de Citrus sinensis, valores superiores (50\%) a los reportados por Perera et al. (2011) en la evaluación de la eficacia de fungicidas naturales y químicos en el control de enfermedades postcosecha sobre distintas variedades de papaya. Estos resultados podrían atribuirse a la concentración de $4 \%$ de aceite y a la calidad de la muestra vegetativa, lo mismo ocurrió en el caso de la inhibición del hongo $C$. gloeosporioides alterando su viabilidad en $100 \%$ con el uso de Citrus sinensis, valores superiores a los reportados en el estudio de propiedad fungistática in vitro de propóleos sobre tres aislamientos de Colletotrichum gloeosporioides (30\% de inhibición) (Pineda et al., 2010). 
Cuadro 2. Porcentajes de inhibición del crecimientos radial y número de esporas

\begin{tabular}{lrrrr}
\hline \multirow{2}{*}{$\begin{array}{c}\text { Tratamientos } \\
\mathbf{A}^{*} \mathbf{B}^{*} \mathbf{C}\end{array}$} & $\begin{array}{r}\text { Inhibición del Crecimiento Radial } \\
(\%)\end{array}$ & \multicolumn{2}{c}{$\begin{array}{c}\text { Inhibición de número de esporas } \\
(\%)\end{array}$} \\
\cline { 2 - 5 } & $\begin{array}{c}\text { Rhizopus } \\
\text { stolonifer }\end{array}$ & $\begin{array}{c}\text { Colletotrichum } \\
\text { gloeosporioides }\end{array}$ & $\begin{array}{c}\text { Rhizopus } \\
\text { stolonifer }\end{array}$ & $\begin{array}{c}\text { Colletotrichum } \\
\text { gloeosporioides }\end{array}$ \\
\hline $\mathrm{a}_{0} \mathrm{~b}_{0} \mathrm{c}_{0}$ & $100.00 \mathrm{c}$ & $100.00 \mathrm{c}$ & $99.76 \mathrm{e}$ & $100.00 \mathrm{~g}$ \\
$\mathrm{a}_{0} \mathrm{~b}_{0} \mathrm{c}_{1}$ & $100.00 \mathrm{c}$ & $100.00 \mathrm{c}$ & $100.00 \mathrm{e}$ & $100.00 \mathrm{~g}$ \\
$\mathrm{a}_{0} \mathrm{~b}_{0} \mathrm{c}_{2}$ & $100.00 \mathrm{c}$ & $100.00 \mathrm{c}$ & $100.00 \mathrm{e}$ & $100.00 \mathrm{~g}$ \\
$\mathrm{a}_{0} \mathrm{~b}_{1} \mathrm{c}_{0}$ & $100.00 \mathrm{c}$ & $100.00 \mathrm{c}$ & $95.79 \mathrm{~d}$ & $95.35 \mathrm{~d}$ \\
$\mathrm{a}_{0} \mathrm{~b}_{1} \mathrm{c}_{1}$ & $100.00 \mathrm{c}$ & $100.00 \mathrm{c}$ & $95.60 \mathrm{~d}$ & $98.79 \mathrm{f}$ \\
$\mathrm{a}_{0} \mathrm{~b}_{1} \mathrm{c}_{2}$ & $100.00 \mathrm{c}$ & $100.00 \mathrm{c}$ & $91.26 \mathrm{c}$ & $96.24 \mathrm{e}$ \\
$\mathrm{a}_{1} \mathrm{~b}_{0} \mathrm{c}_{0}$ & $100.00 \mathrm{c}$ & $100.00 \mathrm{c}$ & $99.76 \mathrm{e}$ & $100.00 \mathrm{~g}$ \\
$\mathrm{a}_{1} \mathrm{~b}_{0} \mathrm{c}_{1}$ & $100.00 \mathrm{c}$ & $100.00 \mathrm{c}$ & $100.00 \mathrm{e}$ & $100.00 \mathrm{~g}$ \\
$\mathrm{a}_{1} \mathrm{~b}_{0} \mathrm{c}_{2}$ & $100.00 \mathrm{c}$ & $100.00 \mathrm{c}$ & $99.76 \mathrm{e}$ & $100.00 \mathrm{~g}$ \\
$\mathrm{a}_{1} \mathrm{~b}_{1} \mathrm{c}_{0}$ & $0.56 \mathrm{a}$ & $52.54 \mathrm{a}$ & $72.51 \mathrm{~b}$ & $50.30 \mathrm{c}$ \\
$\mathrm{a}_{1} \mathrm{~b}_{1} \mathrm{c}_{1}$ & $42.78 \mathrm{~b}$ & $75.97 \mathrm{~b}$ & $64.38 \mathrm{a}$ & $24.49 \mathrm{a}$ \\
$\mathrm{a}_{1} \mathrm{~b}_{1} \mathrm{c}_{2}$ & $100.00 \mathrm{c}$ & $100.00 \mathrm{c}$ & $91.49 \mathrm{c}$ & $28.03 \mathrm{~b}$ \\
\hline
\end{tabular}

Medias con una letra común, no son significativamente diferentes (Tukey, $\mathrm{p}>0.05$ )

Cuadro 3. Comparación de la actividad inhibitoria del crecimiento radial y el número de esporas de hongos del Aceite esencial Citrus sinensis (naranja) frente al aceite esencial de Citrus nobilis (mandarina) en control de Rhizopus Stolonifer y Colletotrichum gloeosporioides (Papaya)

\begin{tabular}{lcccc}
\hline \multirow{2}{*}{ Niveles estudiados } & \multicolumn{2}{c}{ Rhizopus stolonifer } & \multicolumn{2}{c}{ Colletotrichum gloeosporioides } \\
\cline { 2 - 5 } & $\begin{array}{c}\text { Inhibición } \\
\text { Crecimiento radial } \\
(\%)\end{array}$ & $\begin{array}{c}\text { Inhibición de } \\
\text { esporas } \\
(\%)\end{array}$ & $\begin{array}{c}\text { Inhibición } \\
\text { Crecimiento radial } \\
(\%)\end{array}$ & $\begin{array}{c}\text { Inhibición de } \\
\text { esporas } \\
(\%)\end{array}$ \\
\hline C. sinensis (Naranja) & $100.00 \mathrm{~b}$ & $97.06 \mathrm{~b}$ & $100.00 \mathrm{~b}$ & $98.39 \mathrm{~b}$ \\
C. nobilis (Mandarina) & $75.23 \mathrm{a}$ & $87.98 \mathrm{a}$ & $80.91 \mathrm{a}$ & $67.13 \mathrm{a}$ \\
Error Estándar & 1.11 & 0.03 & 1.00 & 0.06 \\
\hline
\end{tabular}

Medias con una letra común, no son significativamente diferentes (Tukey, $\mathrm{p}>0.05$ )

La inhibición de esporas C. gloeosporioides alcanzó un $98.39 \%$ (Citrus sinensis), valor ligeramente inferior al $100 \%$ alcanzado por Landero et al. (2013) en su investigación de potencial antifúngico de extractos de cuatro especies vegetales sobre el crecimiento de Colletotrichum gloeosporioides en papaya (Carica papaya) en poscosecha, estos valores podría atribuirse a varios factores, como las condiciones del ambiente microbiológico, o posiblemente, factores ambientales del sitio de trabajo, que en este caso no fueron controlados como factor determinante en este estudio, a pesar de trabajar en un ambiente regulado (acondicionado).

$\mathrm{Al}$ respecto, los aceites esenciales a partir de hojas y pericarpio de los cítricos estudiados, en el crecimiento radial de $R$. stolonifer se evidenció 100\% (hojas) de inhibición, valor superior al 94\% de inhibición reportado por Barrera y Bautista (2008) en la investigación de actividad antifúngica de polvos, extractos y fracciones de Cestrum nocturnum L. sobre el crecimiento micelial de Rhizopus stolonifer.

En la inhibición de esporas de $R$. stolonifer se logró 99.88\% con aceite obtenido de las hojas (Cuadro 4), valor superior al 88\% obtenidos por Angulo et al. (2009) en la extracción de semilla de Swietenia humilis Zucc con actividad antifúngica de $R$. stolonifer. Mientras, en la inhibición del crecimiento radial del hongo del género $C$. gloeosporioides se obtuvo 100\% (hojas), resultados iguales a los reportados por Prapassom et al. (2012) en el efecto de extracto crudo de hojas contra Colletotrichum gloeosporioides; y en el porcentaje de inhibición del esporas del hongo del género C. gloeosporioides tambien se obtuvo el $100 \%$ con aceite extraído de las hojas, superiores a los reportados por Ademe et al. (2013) quienes publicaron $88.70 \%$ en la evaluación de la actividad antifúngica de extractos de plantas contra la antracnosis (Colletotrichum gloeosporioides) de la papaya.

En lo referente a las concentraciones de aceites (Factor 
Cuadro 4. Valoración de la Actividad inhibitoria en crecimiento radial y número de esporas de hongos. Comparación de aceites provenientes de las hojas frente al aceite de pericarpio

\begin{tabular}{lcccc}
\hline Niveles & \multicolumn{2}{c}{ Rhizopus stolonifer } & \multicolumn{2}{c}{ Colletotrichum gloeosporioides } \\
\cline { 2 - 5 } estudiados & $\begin{array}{c}\text { Inhibición } \\
\text { Crecimiento radial } \\
(\%)\end{array}$ & $\begin{array}{c}\text { Inhibición de } \\
\text { esporas } \\
\mathbf{( \% )}\end{array}$ & $\begin{array}{c}\text { Inhibición } \\
\text { Crecimiento radial } \\
\mathbf{( \% )}\end{array}$ & $\begin{array}{c}\text { Inhibición de } \\
\text { esporas } \\
\mathbf{( \% )}\end{array}$ \\
\hline Hojas & $100.00 \mathrm{~b}$ & $99.88 \mathrm{~b}$ & $100.00 \mathrm{~b}$ & $100.00 \mathrm{~b}$ \\
Pericarpio & $75.23 \mathrm{a}$ & $85.16 \mathrm{a}$ & $88.08 \mathrm{a}$ & $65.53 \mathrm{a}$ \\
Error Estándar & 1.11 & 0.03 & 1.00 & 0.06 \\
\hline
\end{tabular}

Medias con una letra común, no son significativamente diferentes (Tukey, $\mathrm{p}>0.05$ )
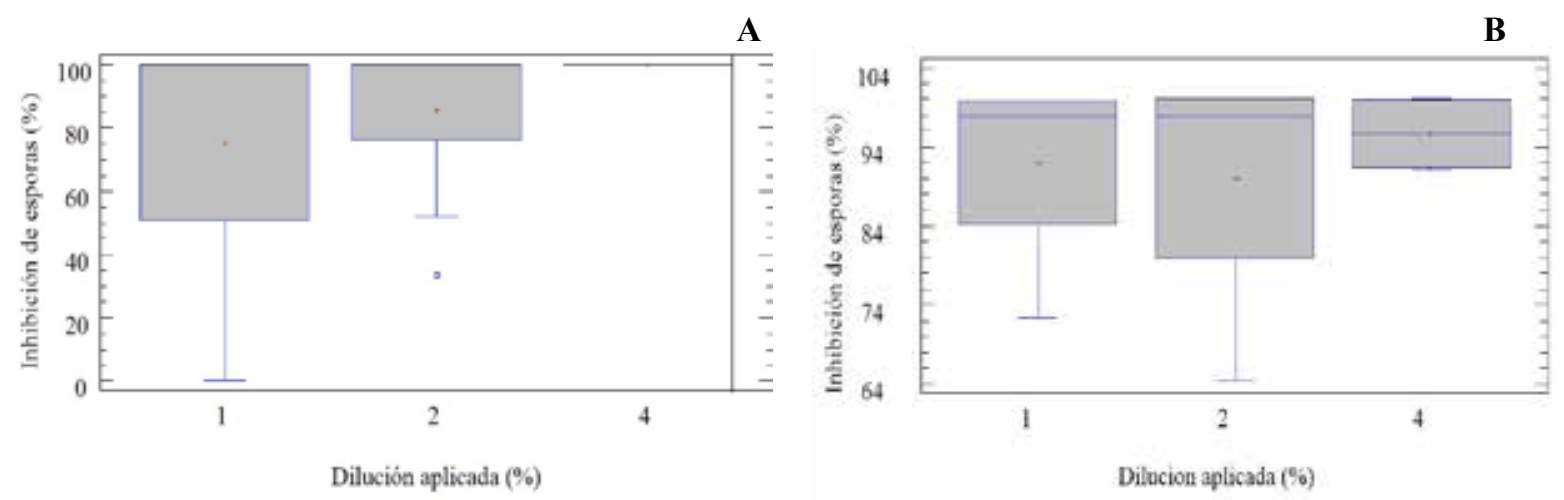

Figura 1. Concentraciones de aceites esenciales $(1 \%, 2 \%, 4 \%)$ en cepas $R$. stolonifer. A) Porcentaje de inhibición del crecimiento radial $(\mathrm{c} 0=\mathbf{7 5}, 13 \%, \mathrm{c} 1=\mathbf{8 5 , 6 9 \%}$ y $\mathrm{c} 2=\mathbf{1 0 0 \%})$; B) Porcentaje de inhibición del número de esporas (c1=89.99\%, c0=91.95\% y c2=95.62\%)

A

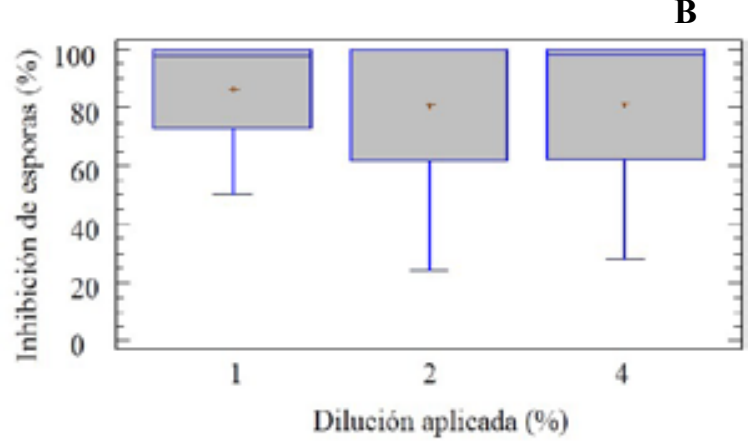

Figura 2. Concentraciones de aceites esenciales $(1 \%, 2 \%, 4 \%)$ en cepas $C$. gloeosporioides. A) Porcentaje de inhibición del crecimiento radial $(\mathrm{c} 0=88.13 \%, \mathrm{c1}=93.99 \%$ y c2=100\%); B) Porcentaje de inhibición del número de esporas $(\mathrm{c} 1=80.81 \%, \mathrm{c} 2=81.06 \%$ y c0 $=86.41 \%)$

C) $(1 \%, 2 \%$ y $4 \%)$ empleados como inhibidores de hongos (Figura 1 y Figura 2), en el crecimiento radial del hongo del género $R$. stolonifer se logró $100 \%$ aplicando $4 \%$ de aceite, igual al reportado por Ronquillo (2007) en la evaluación del potencial antimicrobiano de películas comestibles con aceites esenciales in vitro e in situ. En la evaluación de la inhibición de esporas del hongo R. stolonifer se obtuvo $95.62 \%$ con $4 \%$ de aceite, valores superiores a los obtenido por Tzortzakis y Economakis (2007) en su trabajo de actividad antifúngica del aceite esencial de Lemongrass (Cymbopogon citratus L.) contra hongos patógenos de postcosecha. El crecimiento radial de C. gloeosporioides reveló $100 \%$ de inhibición con el uso de $4 \%$ de solución, este resultado coincide con Guédez et al. (2014) quienes reportaron actividad antifúngica 
del aceite esencial de naranja (Citrus sinensis L.) sobre hongos postcosecha en frutos de lechosa (Carica papaya L.); mientras, Silva et al. (2011) presenta el 61\% de inhibición del crecimiento micelial y la germinación Colletotrichum gloeosporioides aplicando aceite de neem (Azadirachta indica).

\section{Conclusiones}

$\mathrm{E}$ 1 aceite esencial de las hojas y pericarpio de Citrus sinensis aplicado en cepas $R$. stolonifer y $C$. gloeosporioides al 4\% es capaz de inhibir el crecimiento radial y de esporas en el proceso de conservación de papaya de variedad "Maradol"

\section{Agradecimientos}

A la Universidad Técnica Estatal de Quevedo y a la Secretaría Nacional de Educación Superior, Ciencia, Tecnología e Innovación (SENESCYT), Ecuador, por el apoyo a la ejecución de la investigación.

\section{Bibliografía}

Ademe, A., Ayalew, A., \& Woldetsadik, K. (2013). Evaluation of Antifungal Activity of Plant Extracts against Papaya Anthracnose (Colletotrichum gloeosporioides). Plant Pathology \& Microbiology, 4(10).

Angulo, M., Armenta, E., García, R., Carrillo, J., Salazar, E., \& Valdéz, J. (2009). Extractos de Semilla de Swietenia humilis Zucc. con Actividad Antifúngica en Rhizopus stolonifer (Ehrenb.:Fr.) Vuill. Revista Mexican de Fitopatología, 27(2), 84-92.

Barnett, H., \& Hunter, B. (1987). Ilustrated Genera os Imperfect Fungi (4 ed.). NY: Macmillan Publishing Company.

Barrera, L., \& Bautista, S. (2008). Actividad Antifúngica de Polvos, Extractos y Fracciones de Cestrum nocturnum L. Sobre el Crecimiento Micelial de Rhizopus stolonifer (Ehrenb.:Fr.) Vuill. Revista Mexicana de Fitopatología, 26(1), 27-31.

Duarte, Y., Pino, O., \& Martínez, B. (2013). Efecto de cuatro aceites esenciales sobre Fusarium spp. Protección Vegetal, 28(3), 232-235.

Guédez, C., Cañizalez, L., Avendaño, L., Scorza, J., Castillo, C., Olivar, R. (2014). Actividad antifúngica del aceite esencial de naranja (Citrus sinensis L.) sobre hongos postcosecha en frutos de lechosa (Carica papaya L.).
Revista de la Sociedad Venezolana de Microbiología, 81-85.

Landero, N., Nieto, D., Téliz, D., Alatorre, R., Orozco, M., \& Ortiz, C. (2013). Potencial antifúngico de extractos de cuatro especies vegetales sobre el crecimiento de Colletotrichum gloeosporioides en papaya (Carica papaya L.) en poscosecha. Revista Venezolana de Ciencia y Tecnología de Alimentos, 47-62.

Perera, S., Pérez, E., Hernández, J., Lobo, G., López, J., Puerta, M. (2011). Evaluación de la eficacia de fungicidas naturales y químicos en el contol de enfermedades postcosecha sobre ditintas variedades de papaya (II).

Pineda, J., Principal, J., Barrios, C., Milla , D., Solano, Y., \& Gil, E. (2010). Propiedad fungistática in vitro de propóleos sobre tres aislamientos de Colletotrichum gloeosporioides. Bioline.org, 28(1), 83-91.

Prapassom, B., Piyarat, N., Paweena, R., \& Angsuman, C. (2012). Effect of Crude Leaf Extract on Colletotrichum gloeosporioides (Penz.) Sacc. Hindawi, 6.

Rodríguez Lacherre, M., \& Veneros Terrones, R. (2011). Control biológico de Trichoderma harzianum RIFAI sobre hongos patógenos de frutos poscosecha de Carica papaya procedente de zonas de distribución del distrito Trujillo (Perú). Revista de la Facultad de Ciencias Biológicas.

Ronquillo De Jesús, E. (2007). Evaluación del potencial antimicrobiano de películas comestibles con aceites esenciales in vitro e in situ. División CBS, Unidad Iztapalapa. Mexico D.F: Universidad Autónoma Metropolitana. Posgrado en Biotecnología.

Silva, R., Pereira, R., \& Nakano, M. (2011). Inibição do crescimento micelial e germinação de Colletotrichum gloeosporioides na seringueira pelo óleo de neem (Azadirachta indica). Dialnet, 8(1), 295-303.

Suárez, M., Mendoza, I., Monroy, J., De la Cruz, J., Ángulo, O., \& Gónzalez, O. (2013). Aislamiento, identificación y sensibilidad a antifúngicos de hongos fitopatógenos de papaya cv. Maradol (Carica papaya L.). Revista Iberoamericana de Tecnología Postcosecha, 14(2), 115124.

Tzortzakis, N., \& Economakis, C. (2007). Antifungal activity of lemongrass (Cypopogon citratus L.) essential oil against key postharvest pathogens. Elseiver Ltd.

Von-Arx, J. (1981). The genera of fungi sporulating in pure culture, Stratus and Cramer. Alemania. 